\title{
TAKING TILTING MODULES FROM THE POSET OF SUPPORT TILTING MODULES
}

\author{
RYOICHI KASE
}

\begin{abstract}
C. Ingalls and H. Thomas defined support tilting modules for path algebras. From $\tau$-tilting theory introduced by T. Adachi, O. Iyama and I. Reiten, a partial order on the set of basic tilting modules defined by D. Happel and L. Unger is extended as a partial order on the set of support tilting modules. In this paper, we study a combinatorial relationship between the poset of basic tilting modules and basic support tilting modules. We will show that the subposet of tilting modules is uniquely determined by the poset structure of the set of support tilting modules.
\end{abstract}

\section{INTRODUCTION}

Tilting theory first appeared in an article by Brenner and Butler [BB]. In that article the notion of a tilting module for finite dimensional algebras was introduced. Tilting theory now appears in many areas of mathematics, for example algebraic geometry, theory of algebraic groups and algebraic topology. Let $T$ be a tilting module for a finite dimensional algebra $\Lambda$ and let $B=\operatorname{End}_{A}(T)$. Then Happel showed that the two bounded derived categories $\mathcal{D}^{\mathrm{b}}(A)$ and $\mathcal{D}^{\mathrm{b}}(B)$ are equivalent as triangulated category [H]. Therefore, classifying tilting modules is an important problem.

Tilting mutation introduced by Riedtmann and Schofield is an approach to this problem. It is an operation which gives a new tilting module from given one by replacing an indecomposable direct summand. They also introduced a tilting quiver whose vertices are (isomorphism classes of) basic tilting modules and arrows correspond to mutations. Happel and Unger defined a partial order on the set of basic tilting modules and showed that the tilting quiver coincides with the Hasse quiver of this poset. However, tilting mutation is often impossible depending on a choice of an indecomposable direct summand. Support $\tau$-tilting modules introduced by Adachi, Iyama and Reiten are generalization of tilting modules. They showed that a mutation (resp. a partial order) on the set of (isomorphism classes of) basic tilting modules is extended as an operation (resp. a partial order) on the set of (isomorphism classes of) support $\tau$-tilting modules. They also showed that support $\tau$-tilting mutation has following nice properties:

- Support $\tau$-tilting mutation is always possible.

- Support $\tau$-tilting quiver coincides with the Hasse quiver of the poset of support $\tau$-tilting modules.

Notation. Throughout this paper, let $\Lambda$ be a finite dimensional algebra over an algebraically closed field $k$.

(1) We always assume that $\Lambda$ is basic and indecomposable.

(2) We denote by $\bmod \Lambda$ the category of finitely generated right $\Lambda$-modules.

Key words and phrases. tilting modules, tilting mutations, representations of quivers,

2010 Mathematics Subject Classification. Primary 16G20; Secondary 16D80, 16G30. 
(3) We denote by $\tau$ the Auslander-Reiten translation of $\bmod \Lambda$.

(4) A module means a finitely generated right module.

(5) We denote by tilt $(\Lambda)$ (respectively, s-tilt $(\Lambda), \mathrm{s} \tau$-tilt $(\Lambda))$ the (partially ordered) set of (isomorphism classes of) basic tilting (respectively, support tilting, support $\tau$ tilting) $\Lambda$-modules (see Section 2 below for the definition).

In this paper, we consider a combinatorial relationship between $\mathrm{s} \tau$-tilt $(\Lambda)$ and $\operatorname{tilt}(\Lambda)$. Since tilting or support $\tau$-tilting mutation is introduced for the aim of obtaining many tilting modules, the following is an interesting question.

Question 1.1. Is the set of tilting modules tilt $(\Lambda)$ uniquely determined by the posetstructure of $\mathrm{s} \tau$-tilt $(\Lambda)$ ?

The main result of this paper is the following.

Theorem 1.2. Let $\Lambda$ and $\Gamma$ be two finite dimensional basic hereditary algebras. If $\rho$ is a poset isomorphism from $\mathrm{s} \tau$-tilt $(\Lambda)$ to $\mathrm{s} \tau$-tilt $(\Gamma)$, then the restriction of $\rho$ to $\operatorname{tilt}(\Lambda)$ induces a poset isomorphism

$$
\left.\rho\right|_{\operatorname{tilt}(\Lambda)}: \operatorname{tilt}(\Lambda) \simeq \operatorname{tilt}(\Gamma)
$$

\section{Preliminaries}

2.1. Tilting modules. In this subsection we recall the definition of tilting modules. For a module $M$, we denote by $|M|$ the number of non-isomorphic indecomposable direct summand of $M$.

Definition 2.1. A $\Lambda$-module $M$ is said to be a partial tilting module if it satisfies following conditions.

(i) $\operatorname{pd} T \leq 1$.

(ii) $\operatorname{Ext}_{\Lambda}^{1}(T, T)=0$.

If partial tilting module $T$ satisfies $|M|=|\Lambda|$, then we call $T$ a tilting module. The set of non-isomorphic basic tilting modules of $\Lambda$ is denoted by tilt $\Lambda$

For a $\Lambda$-module $M$, we put $M^{\perp_{1}}:=\left\{X \in \bmod \Lambda \mid \operatorname{Ext}_{\Lambda}^{1}(M, X)=0\right\}$.

Definition-Theorem 2.2. [HU1] Let $T_{1}$ and $T_{2}$ be two tilting modules. We write $T_{1} \leq T_{2}$ if $T_{1}^{\perp_{1}} \subset T_{2}^{\perp_{1}}$. Then $\leq$ defines a partial order on $\operatorname{tilt}(\Lambda)$.

It is known that if $T$ is a tilting module, then $X$ is in $T^{\perp_{1}}$ if and only if $X$ is a factor module of finite direct sums of copies of $T$. Therefore we have the following [HU2] :

$$
T \geq T^{\prime} \Leftrightarrow \operatorname{Ext}_{\Lambda}^{1}\left(T, T^{\prime}\right)=0 .
$$

2.2. Support $\tau$-tilting modules. The notion of support $\tau$-tilting modules which were introduced in $\mathrm{AIR}$, is a generalization of that of tilting modules.

Let us recall the definition of support $\tau$-tilting modules.

Definition 2.3. AIR Let $M$ be a $\Lambda$-module and $P$ be a projective $\Lambda$-module.

(1) $M$ is said to be a $\tau$-rigid module if it satisfies $\operatorname{Hom}_{\Lambda}(M, \tau M)=0$.

(2) $(M, P)$ is said to be a $\tau$-rigid pair if $M$ is a $\tau$-rigid module and $\operatorname{Hom}_{\Lambda}(P, M)=0$.

(3) $(M, P)$ is called a support $\tau$-tilting pair if it is a $\tau$-rigid pair with $|M|+|P|=|\Lambda|$. We then call $M$ a support $\tau$-tilting module. The set of non-isomorphic basic support $\tau$-tilting modules of $\Lambda$ is denoted by s $\tau$-tilt $\Lambda$. 
We note that if $M \in \mathrm{s} \tau$-tilt $(\Lambda)$, then there is a unique (up to isomorphism) basic projective module $P$ such that $(M, P)$ is a support $\tau$-tilting pair [AIR].

Definition-Theorem 2.4. AIR] Let $(M, P)$ and $\left(M^{\prime}, P^{\prime}\right)$ be two support $\tau$-tilting pair. We write $M \leq M^{\prime}$ if $\operatorname{Hom}_{\Lambda}\left(M, \tau M^{\prime}\right)=0$ and add $P^{\prime} \subset$ add $P$. Then $\leq$ defines a partial order on $\mathrm{s} \tau$-tilt $(\Lambda)$.

We call $(N, U)$ an almost complete support $\tau$-tilting pair if $(N, U)$ is a $\tau$-rigid pair with $|N|+|U|=|\Lambda|-1$.

Theorem 2.5. AIR.

(1) Let $(N, U)$ be a basic almost complete support $\tau$-tilting pair. Then $(N, U)$ is a direct summand of exactly two support $\tau$-tilting pairs.

(2) Let $(M, P)$ and $\left(M^{\prime}, P^{\prime}\right)$ be two support $\tau$-tilting pair. Then there is an edge $M-M^{\prime}$ in the underlying graph of the Hasse quiver of $\mathrm{s} \tau$-tilt $(\Lambda)$ if and only if there exists basic almost complete support $\tau$-tilting pair $(N, U)$ such that $(N, U)$ is a direct summand of $(M, P)$ and $\left(M^{\prime}, P^{\prime}\right)$.

For a basic $\tau$-rigid module $U$, we denote by s $\tau$-tilt ${ }_{U}(\Lambda):=\{T \in \mathrm{s} \tau$-tilt $(\Lambda) \mid U \in$ add $T\}$.

Theorem 2.6. [J] Let $U$ be a basic partial $\tau$-tilting module. Then there is a finite dimensional algebra $C$ with $|C|=|\Lambda|-|U|$ such that $\mathbf{s} \tau$-tilt $U(\Lambda) \simeq \mathrm{s} \tau$-tilt $(C)$.

2.3. Hereditary case. Let $Q$ be a finite connected acyclic quiver. We denote by $Q_{0}$ (resp. $Q_{1}$ ) the set of vertices (resp. arrows) of $Q$. From now on, we assume that $\Lambda$ is a path algebra $k Q$. In this paper, for any paths $w: a_{0} \stackrel{\alpha_{7}}{\rightarrow} a_{1} \stackrel{\alpha_{2}}{\rightarrow} \cdots \stackrel{\alpha_{r}}{\rightarrow} a_{r}$ and $w^{\prime}: b_{0} \stackrel{\beta_{1}}{\rightarrow} b_{1} \stackrel{\beta_{2}}{\rightarrow} \cdots \stackrel{\beta_{s}}{\rightarrow} b_{s}$ in $Q$, the product is defined by

$$
w \cdot w^{\prime}:= \begin{cases}a_{0} \stackrel{\alpha_{7}}{\rightarrow} a_{1} \stackrel{\alpha_{2}}{\rightarrow} \cdots \stackrel{\alpha_{r}}{\rightarrow} a_{r}=b_{0} \stackrel{\beta_{1}}{\rightarrow} b_{1} \stackrel{\beta_{2}}{\rightarrow} \cdots \stackrel{\beta_{s}}{\rightarrow} b_{s} & \text { if } a_{r}=b_{0} \\ 0 & \text { if } a_{r} \neq b_{0},\end{cases}
$$

in $k Q$. For a module $M \in \bmod \Lambda$, we denote by $Q(M)$ the full subquiver of $Q$ with $Q(M)_{0}=\operatorname{supp}(M):=\left\{a \in Q_{0} \mid(\underline{\operatorname{dim}} M)_{a}>0\right\}$. By definition, we can regard $M$ as a sincere $k Q(M)$-module.

Definition 2.7. [AIR, IT] A $\Lambda$-module $M$ is said to be a support tilting module if $M$ is a tilting $k Q(M)$-module.

Since $\Lambda$ is a finite dimensional hereditary algebra, we have $\mathrm{s} \tau$-tilt $(\Lambda)=$ s-tilt $(\Lambda)$ (see $[\mathrm{AIR}]$ ) and the partial order on s-tilt $(\Lambda)$ is defined as follows:

$$
M \geq M^{\prime} \Leftrightarrow \operatorname{Ext}_{\Lambda}^{\prime}\left(M, M^{\prime}\right)=0 \text { and } Q\left(M^{\prime}\right)_{0} \subset Q(M)_{0} . \quad\left(M, M^{\prime} \in \text { s-tilt }(\Lambda)\right)
$$

Theorem 2.8. [J] Let $N$ be a basic partial tilting module. Then there is a finite dimensional hereditary algebra $C$ with $|C|=|\Lambda|-|N|$ such that $\mathrm{s}-\operatorname{tilt}_{N}(\Lambda) \simeq \mathrm{s}$-tilt $(C)$.

Let $M \in \bmod \Lambda$ and let $P$ be a projective $\Lambda$-module. We set

$$
\mathrm{T}_{\Lambda}(M, P)=\mathrm{T}(M, P):=\left(\tau M \oplus \nu(P), M_{\mathrm{pr}}\right)
$$

where $\nu$ is the Nakayama functor and $M_{\mathrm{pr}}$ is a maximal projective direct summand. We also set

$$
\mathrm{T}^{-}(M, P):=\left(\tau^{-} M \oplus P, \nu^{-} M_{\mathrm{in}}\right)
$$


where $M_{\text {in }}$ is a maximal injective direct summand. Note that

$$
\mathrm{TT}^{-}(M, P)=(M, P)=\mathrm{T}^{-} \mathrm{T}(M, P) .
$$

Lemma 2.9. $\mathrm{AIR}(M, P)$ is a support tilting pair if and only if $\mathrm{T}(M, P)$ is a support tilting pair. In particular $\mathrm{T}$ and $\mathrm{T}^{-}$induces a graph automorphism

$$
G(\operatorname{s-tilt}(\Lambda)) \simeq G(\text { s-tilt }(\Lambda))
$$

where $G($ s-tilt $\Lambda)$ is the underlying graph of the Hasse quiver of s-tilt $(\Lambda)$.

Example 2.10. The following are well-known example. Let $\vec{\Delta}$ be a 2-point acyclic quiver with $\vec{\Delta}_{0}=\{1,2\}$.

(1) If $\vec{\Delta}$ is not connected then s-tilt $(k \vec{\Delta})$ is as follows:

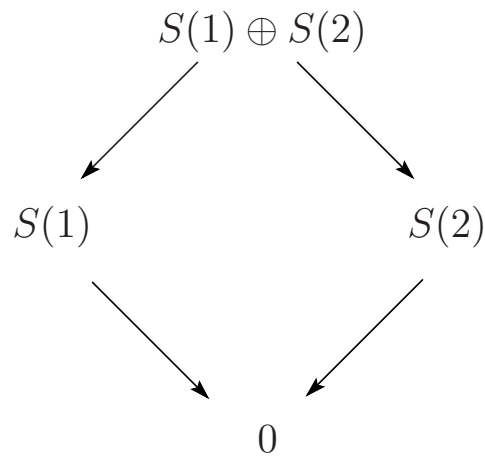

(2) If there is a unique arrow from 1 to 2 . Then s-tilt $(k \vec{\Delta})$ is as follows:

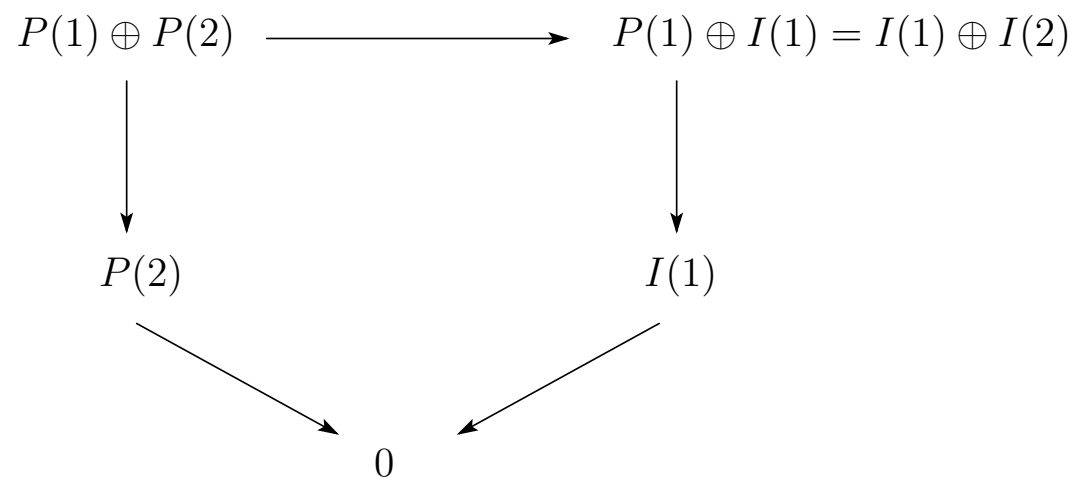

(3) If there are at least two arrows from 1 to 2 . Then s-tilt $(k \vec{\Delta})$ is as follows: 


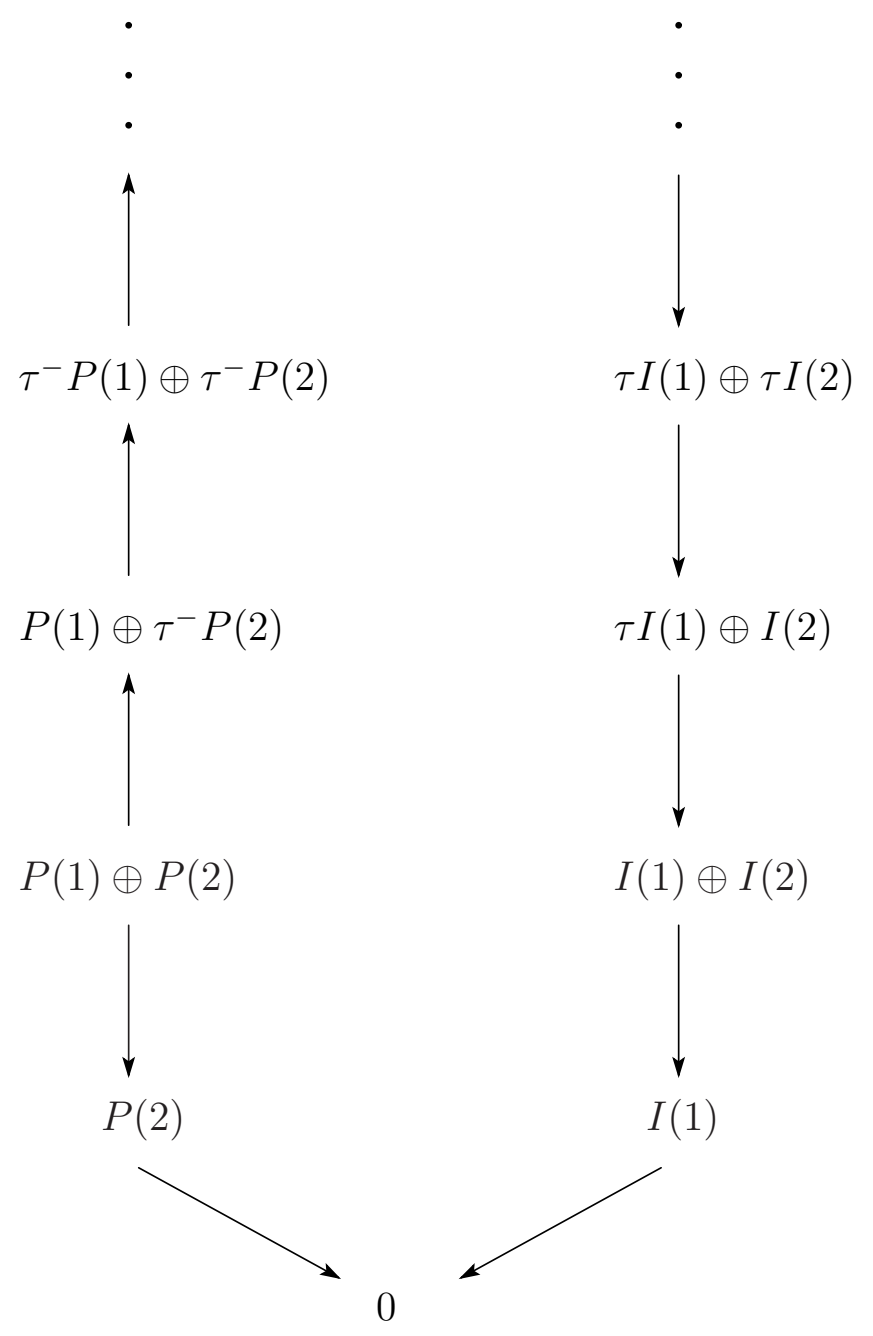

\section{Proof of Theorem 1.2}

In this section, we give a proof of Theorem 1.2, Let $Q$ be a finite connected quiver, $\Lambda:=k Q$. For any $i \in \mathbb{Z}_{\geq 0}$, we define a full subquiver $Q(i)$ of $Q$ as follows:

- $Q(0)_{0}:=\emptyset$

- $Q(i)_{0}:=Q_{i-1} \cup\left\{a \in Q_{0} \mid a\right.$ is a source of $\left.Q \backslash Q_{i-1}\right\}$.

Let $m \in \mathbb{Z}_{\geq 1}$ be a minimum integer satisfying $Q(m)=Q$. For any $i \leq m$, we set $I_{0}:=0$ and $I_{i}:=\bigoplus_{a \in Q(i)_{0}} I(a)$. Note that we can regard $I_{i} \in \bmod \Lambda$ as a basic injective tilting module of $k Q(i)$.

3.1. Neighbours of $I_{i}$. For a support tilting module $M$, we denote by $e(M)$ the set of direct predecessors of $M$ and $s(M)$ the set of direct successors of $M$. If $I$ is an injective support tilting module, then we have

$$
e(I)=\left\{I_{Q(I) \cup\{a\}} \mid a \in Q_{0} \backslash Q(I)_{0}\right\} \sqcup\left\{I / I(a) \oplus \tau_{Q(I)} S(a) \mid a \text { is not a sink of } Q(I)\right\},
$$

where $I_{Q(I) \cup\{a\}}$ be a injective tilting module of $k(Q(I) \cup\{a\})$. We also have

$$
s(I)=\{I / I(a) \mid a \text { is a sink of } Q(I)\} .
$$


It is easy to check that $I_{Q(I) \cup\{a\}}$ is injective in $\bmod \Lambda$ if and only if $a$ is a source of $Q \backslash Q(I)$. In particular we obtain the following:

$$
e\left(I_{i}\right)=e_{1}(i) \sqcup e_{2}(i) \sqcup e_{3}(i), s\left(I_{i}\right)=\left\{I_{i} / I(d) \mid d \text { is a sink of } Q(i)\right\},
$$

where

$$
\begin{aligned}
& e_{1}(i):=\left\{I_{i} \oplus I(a) \mid a \in Q(i+1)_{0} \backslash Q(i)_{0}\right\} \\
& e_{2}(i):=\left\{I_{\left.Q_{i} \cup\{b\} \mid b \in Q_{0} \backslash Q(i+1)_{0}\right\}}\right. \\
& e_{3}(i):=\left\{I_{i} / I(c) \oplus \tau_{Q(i)} S(c) \mid c \in Q(i)_{0} \text { is not a sink of } Q(i)\right\} .
\end{aligned}
$$

3.2. Determining injective predecessors. In this subsection we show that the set of injective predecessors $e_{1}(i)$ of $I_{i}$ is determined by poset structure of s-tilt $(\Lambda)$. First we consider the case $i=0$.

Lemma 3.1. Let $a, b \in Q_{0}$. Then there is an arrow $a \rightarrow b$ in $Q$ if and only if there are $X \in e(S(a))$ and $Y \in e(S(b))$ such that $X<Y$.

Proof. Let $\vec{\Delta}$ be a full subquiver of $Q$ with $\vec{\Delta}_{0}=\{a, b\}$. Assume that there is an arrow $a \rightarrow b$ in $Q$. We denote by $P:=P_{\vec{\Delta}}\left(\right.$ resp. $\left.I:=I_{\vec{\Delta}}\right)$ a basic projective (resp. injective) tilting $k(\vec{\Delta})$-module. We note that $I \in e(S(a))$ and $P \in e(S(b))$ with $I<P$.

Next we assume that there are $X \in e(S(a))$ and $Y \in e(S(b))$ such that $X<Y$. Since $Y>S(a)$ and $Y \in e(S(b)), Y$ must be tilting $k(\vec{\Delta})$-module with $S(b) \in \operatorname{add} Y$. On the other hand, $Y>X \in e(S(a))$ implies that $X$ is $k(\vec{\Delta})$-module with $S(a) \in$ add $X$. If $\vec{\Delta}$ is not connected, then $X=S(a) \oplus S(b)=Y$. Therefore we have $\vec{\Delta}$ is connected. Suppose that there is an arrow $a \leftarrow b$. Then we have $\operatorname{Ext}_{\Lambda}^{1}(S(b), S(a)) \neq 0$. Since $S(a) \in \operatorname{add} X$, $S(b) \in \operatorname{add} Y$ and $X<Y$, we reach a contradiction. Thus there is an arrow $a \rightarrow b$.

Lemma 3.1 shows that $S \in e(0)$ is injective if and only if for any $S^{\prime}, X, Y \in$ s-tilt $(\Lambda)$ with $S^{\prime} \in e(0), X \in e\left(S^{\prime}\right)$ and $Y \in e(S)$, we have $X \nless Y$. In particular, the set of injective predecessors $e_{1}(0)$ of 0 is determined by poset-structure of s-tilt $(\Lambda)$. We now assume $i>0$.

Lemma 3.2. Let $T \in e_{2}(i)$. Then there are $X, Y, Z \in \operatorname{s-tilt}(\Lambda)$ such that $X \in e(T)$, $Y \in e\left(I_{i}\right), Z \in e(Y)$ and $X>Z$.

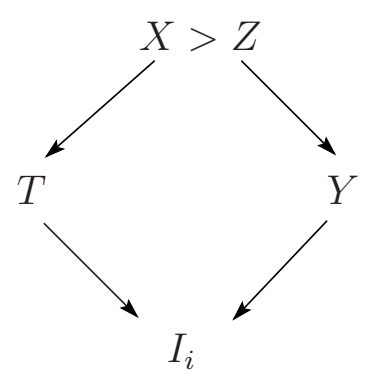

Proof. By definition, there is a vertex $b \in Q_{0} \backslash Q(i+1)_{0}$ such that $T=I_{i} \oplus B=I_{Q(i) \cup\{b\}}$. Then there is a vertex $x \in Q_{0} \backslash Q(i)$ such that $x \rightarrow b$. We note that $I_{Q(i) \cup\{x\}}$ is injective in $\bmod k(Q(i) \cup\{x, b\})$. We also note that there is an indecomposable module $X^{\prime} \in \bmod \Lambda$ such that $T \oplus X^{\prime}$ is a tilting $k(Q(i) \cup\{x, b\})$-module. 
We now assume that $T \oplus X^{\prime}=I_{Q(i) \cup\{x, b\}}$ and let $\vec{\Delta}$ be a full subquiver of $Q$ with $\vec{\Delta}_{0}=\{x, b\}$. We put $\Lambda^{\prime}=k(Q(i) \cup\{x, b\})$. Then the underlying graph $G\left(\mathrm{~s}-\mathrm{tilt}_{I_{i}}\left(\Lambda^{\prime}\right)\right)$ of the Hasse quiver of s-tilt $I_{i}\left(\Lambda^{\prime}\right)$ contains

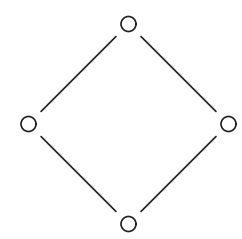

By using Lemma 2.9, we have a graph isomorphism

$$
G\left(\text { s-tilt } _ { I _ { i } } ( \Lambda ^ { \prime } ) \simeq G \left(\mathrm{~T}_{\Lambda^{\prime}}^{-}\left(\mathrm{s}^{-} \text {tilt }_{I_{i}}\left(\Lambda^{\prime}\right)\right)\right.\right.
$$

Note that $\mathrm{T}_{\Lambda^{\prime}}^{-}\left(\right.$s-tilt $\left._{I_{i}}\left(\Lambda^{\prime}\right)\right)=\left\{T \in\right.$ s-tilt $\left.\left(\Lambda^{\prime}\right) \mid Q(T)_{0} \subset\{x, b\}\right\}$. In particular we obtain a graph isomorphism

$$
G\left(\text { s-tilt }_{I_{i}}\left(\Lambda^{\prime}\right)\right) \simeq G(\text { s-tilt }(k \vec{\Delta})) .
$$

Since $\vec{\Delta}$ is a 2-point connected acyclic quiver, $G($ s-tilt $(k \vec{\Delta}))$ has one of the following form (see Example 2.10):

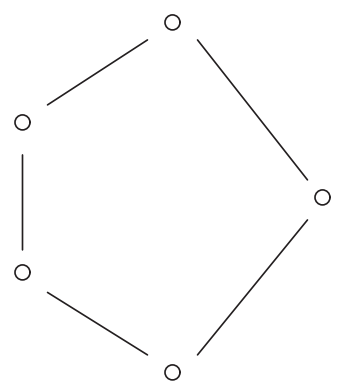

or

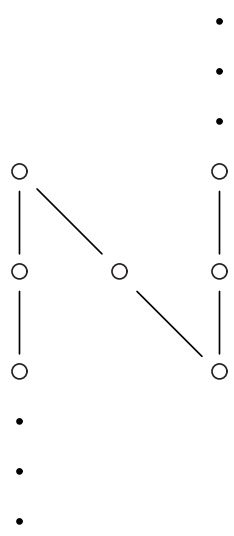

Hence we reach a contradiction.

Therefore $X:=T \oplus X^{\prime}, Y:=I_{Q(i) \cup\{x\}}$ and $Z=I_{Q(i) \cup\{x, b\}}$ satisfy desired property.

Lemma 3.3. Let $T \in e_{1}(i)$. Then for any $X, Y, Z \in$ s-tilt $(\Lambda)$ with $X \in e(T), Y \in e\left(I_{i}\right)$ and $Z \in e(Y)$, we have $X \ngtr Z$.

Proof. Suppose that there are $X, Y, Z \in$ s-tilt $(\Lambda)$ such that

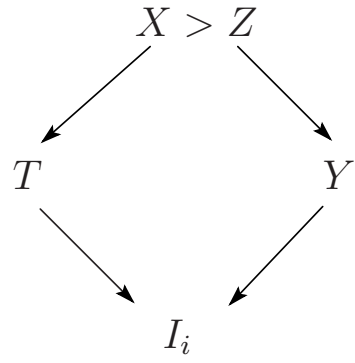

Let $a \in Q(i+1)_{0} \backslash Q(i)_{0}$ with $T=I_{i} \oplus I(a)$. 
First we assume that $Y \in e_{3}(i)$. Then there is a vertex $c \in Q(i)_{0}$ which is not sink of $Q(i)$ such that

$$
Y=I_{i} / I(c) \oplus \tau_{Q(i)} S(c)
$$

In this case, $\tau_{Q(i)} S(c) \in$ add $Z$. Hence we have

$$
X=\left(I_{i} / I(c)\right) \oplus I(a) \oplus \tau_{Q(i) \cup\{a\}} S(c) .
$$

If $Q(Z)=Q(Y)=Q(i)$, then there is a vertex $z \in Q(i)_{0} \backslash\{c\}$ such that $I(z) \notin$ add $Z$. On the other hand, $I(z)$ must be a direct summand of $X$. Since $X>Z$, we have

$$
\operatorname{Ext}_{\Lambda}^{1}(I(z), Z)=0=\operatorname{Ext}_{\Lambda}^{1}(Z, I(z)) .
$$

Hence we obtain $I(Z) \in \operatorname{add} Z$. This is a contradiction. Thus we can assume that $Q(Z)=Q(Y) \cup\{z\}$ for some $z \in Q_{0}$. Then there is an indecomposable module $Z^{\prime} \in \bmod \Lambda$ such that $Z=Y \oplus Z^{\prime} . X>Z$ implies

$$
Q(i) \cup\{z\}=Q(Z) \subset Q(X)=Q(i) \cup\{a\} .
$$

Therefore we obtain $z=a . X>Z$ also implies that

$$
\operatorname{Ext}_{\Lambda}^{1}(I(a), Z)=0=\operatorname{Ext}_{\Lambda}^{1}(Z, I(a)) .
$$

We conclude that $I(a) \in \operatorname{add} Z$. In particular, we have that

$$
Z=Y \oplus I(a) \text {. }
$$

Now $T, X, Z \in \operatorname{tilt}(k(Q(i) \cup\{a\}))$ are complements of almost complete partial tilting $k(Q(i) \cup\{a\})$-module $\left(I_{i} / I(c)\right) \oplus I(a)$. This is a contradiction.

Next we assume that $Y \in e_{2}(i)$. Then there is a vertex $b \in Q_{0} \backslash Q(i+1)_{0}$ such that $Q(Y)=Q(i) \cup\{b\} . X>Y$ implies that $Q(Y) \subset Q(X)$. Thus we obtain

$$
Q(X)=Q(i) \cup\{a, b\}=Q(T) \cup\{b\} \text { and } X=I_{Q(i) \cup\{a, b\}} .
$$

If $Q(X)=Q(Z)$, then we have $X \leq Z$. This is a contradiction. Therefore we can suppose that $Q(Z)=Q(Y)$. Let $B$ be an indecomposable $\Lambda$-module such that $Y=I_{i} \oplus B$. Then $B$ must be a direct summand of $Z$. Therefore we have that $I_{i} \notin$ add $Z$. On the other hand, $X>Z$ implies that $I_{i} \in$ add $Z$. We reach a contradiction.

Therefore $Y$ must be an element of $e_{1}(i)$. Then there is a vertex $a^{\prime} \in Q(i+1)_{0} \backslash Q(i)_{0}$ such that $Y=I_{i} \oplus I\left(a^{\prime}\right)$. In this case, $X>Y$ implies that

$$
X=I_{i} \oplus I(a) \oplus I\left(a^{\prime}\right) .
$$

This implies that $Y \in s(X)$. This contradict to $Y<Z<X$.

Let $T \in e\left(I_{i}\right)$. For any $r \in Z_{\geq 1}$, we set

$$
\mathcal{F}(i, T, r):=\left\{\left(\left(X_{k}\right)_{k \in\{1, \cdots, r\}},\left(T_{k}\right)_{k \in\{1 \cdots, r-1\}},\left(Y_{k}\right)_{k \in\{1, \cdots, r-1\}}\right) \mid(\star)\right\}
$$

where $(\star)$ is the following:

$$
(\star) \begin{cases}\bullet & X_{1} \in s\left(I_{i}\right), X_{k+1} \in s\left(X_{k}\right) \\ \bullet & T_{k} \in e\left(X_{k}\right) \backslash\left\{X_{k-1}\right\} \\ \bullet & Y_{k} \in e\left(T_{k}\right) \\ \bullet & Y_{1} \geq T, Y_{k+1} \geq T_{k}\end{cases}
$$

We let $\mathcal{F}(i, T):=\bigsqcup_{r \geq 1} \mathcal{F}(i, T, r)$. 


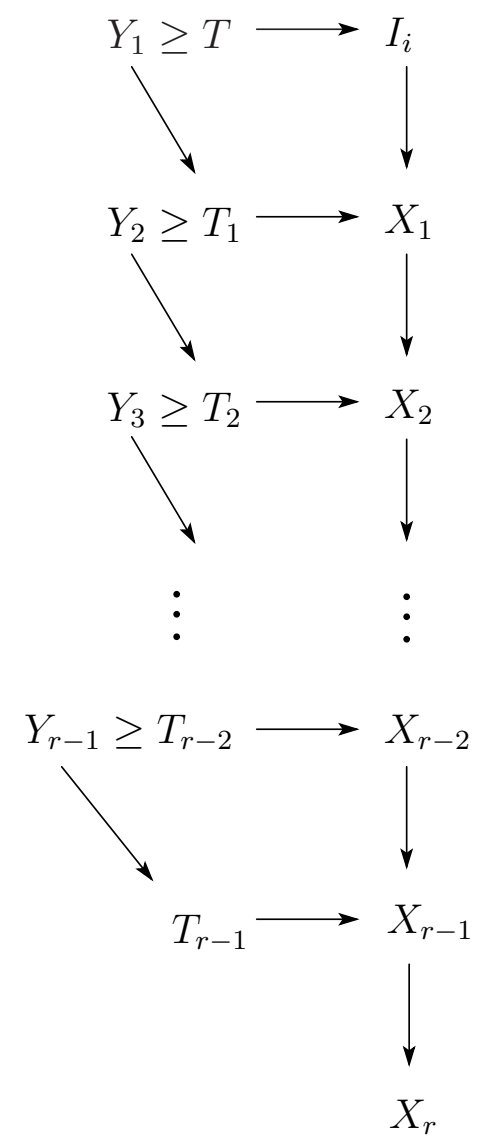

Lemma 3.4. Let $T \in e_{3}(i)$. Then there exists $\left(\left(X_{k}\right),\left(T_{k}\right),\left(Y_{k}\right)\right) \in \mathcal{F}(i, T, r)$ such that for any $T_{r}, Y_{r}$ satisfying $T_{r} \in e\left(X_{r}\right) \backslash\left\{X_{r-1}\right\}$ and $Y_{r} \in e\left(T_{r}\right)$, we have $Y_{r} \nsupseteq T_{r-1}$

Proof. Let $T=T_{i} / I(a) \oplus \tau_{Q(i)} S(a)$. We denote by $\vec{\Delta}(i, a)$ the full subquiver of $Q$ with $\vec{\Delta}(i, a)_{0}:=\left\{x \in Q(i)_{0} \mid x\right.$ is a successor of $\left.a\right\}$.

We define $b_{k} \in Q(i)_{0}\left(k=1,2, \cdots, r:=\# \vec{\Delta}(i, a)_{0}\right)$ as follows:

(1) $b_{1}$ be a sink of $\vec{\Delta}(i, a)$.

(2) $b_{k}$ be a sink of $\vec{\Delta}(i, a) \backslash\left\{b_{1}, \cdots, b_{k-1}\right\}$.

We set

$$
X_{k}:=I_{i} / \bigoplus_{j \leq k} I\left(b_{j}\right) .
$$

We note that $X_{1}$ is a direct successor of $I_{i}$ and $X_{k+1}$ is a direct successor of $X_{k}$. For any $k \in\{1,2, \cdots, r-1\}$, we put

$$
Q(i, k):=Q(i) \backslash\left\{b_{1}, \cdots, b_{k}\right\}
$$

and define $T_{k}$ and $Y_{k}$ as follows:

- $T_{k}:=X_{k} / I_{a} \oplus \tau_{Q(i, k)} S(a)$.

- $Y_{k}$ is a unique direct predecessor of $T_{k}$ with $Q(Y)=Q(i, k-1)$.

We claim that $\left(\left(X_{k}\right),\left(T_{k}\right),\left(Y_{k}\right)\right) \in \mathcal{F}(i, T, r)$. If $r=1$, then the assertion is obvious. Hence we assume $r \geq 2$. Since $T_{k} \in e\left(X_{k}\right) \backslash\left\{X_{k-1}\right\}, Y_{k} \in e\left(T_{k}\right)$, it is sufficient to show 
that

$$
Y_{k+1} \geq T_{k}(k=0,1, \cdots, r-2),
$$

where we put $T_{0}=T$. Without loss of generality, we can assume that $Y_{k+1} \not T_{k}$. Then poset s-tilt $X_{k+1} / I(a)(k Q(i, k))$ contains

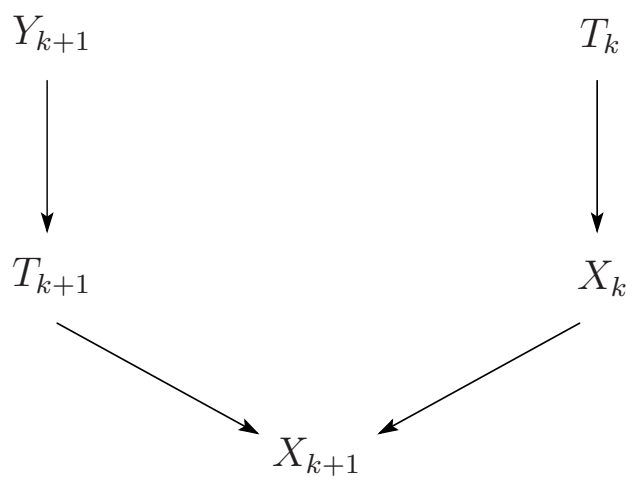

On the other hand, there is a 2-point acyclic quiver $\vec{\Delta}$ such that

$$
\text { s-tilt }_{X_{k+1} / I(a)}(k Q(i, k)) \simeq \mathrm{s} \text {-tilt }(k \vec{\Delta}) .
$$

If $\vec{\Delta}$ is not connected, then \#s-tilt $(k \vec{\Delta})=4$ and this is a contradiction. Therefore we

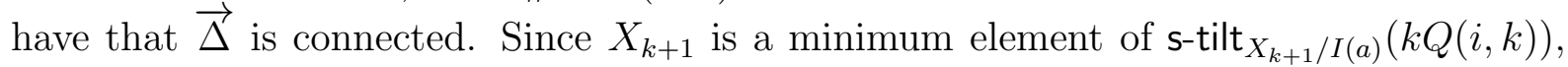
either $Y_{i+1} \geq T_{k}$ or $Y_{k+1}<T_{k}$ hold (see Example 2.10). Since $Q\left(Y_{k+1}\right)=Q(i, k)=Q\left(X_{k}\right)$ and $X_{k}=I_{Q(i, k)}$, we have $Y_{k+1} \geq X_{k}$. If $Y_{k+1}<T_{k}$, then we conclude $X_{k}<Y_{k+1}<T_{k}$ and $T_{k} \in e\left(X_{k}\right)$. We reach a contradiction. Hence we obtain

$$
\left(\left(X_{k}\right),\left(T_{k}\right),\left(Y_{k}\right)\right) \in \mathcal{F}(i, T, r) .
$$

Next we suppose that there are $T_{r}, Y_{r}$ such that

$$
T_{r} \in e\left(X_{r}\right), Y_{r} \in e\left(T_{r}\right) \text { and } Y_{r} \geq T_{r-1} .
$$

Note that $a$ is a sink of $Q(i) \backslash\left\{b_{1}, \cdots, b_{r}\right\}=Q\left(X_{r}\right)$, hence $I(a)$ must be a direct summand of $T_{r}$. Note also that $b_{r} \notin Q\left(T_{r}\right)_{0}$ and $b_{r} \in Q\left(T_{r-1}\right)_{0} \subset Q\left(Y_{r}\right)_{0}$. Therefore we have $T_{r} \in$ add $Y_{r}$. In particular, $I(a)$ is a direct summand of $Y_{r}$. Since $Y_{r} \geq T_{r-1}$ and $I(a)$ is an injective module, we obtain $I(a) \in \operatorname{add} T_{r-1}$. This is a contradiction.

Lemma 3.5. Let $T \in e_{1}(i),\left(\left(X_{k}\right),\left(T_{k}\right),\left(Y_{k}\right)\right) \in \mathcal{F}(i, T, r)$. Then there are $T_{r}, Y_{r}$ such that $T_{r} \in e\left(X_{r}\right)$ and $T_{r-1} \leq Y_{r} \in e\left(T_{r}\right)$.

Proof. Let $T=I_{i} \oplus I(a)$. By definition, there exists $\left(b_{k}\right)_{k=1, \cdots, r} \in Q(i)^{r}$ such that

$$
X_{k}=I_{i} / \bigoplus_{j \leq k} I\left(b_{j}\right)
$$

By using induction, we show that

$$
\text { (*) } Q\left(Y_{k}\right)=Q\left(X_{k}\right) \cup\left\{a, b_{k}\right\} \text { and } Q\left(T_{k}\right)=Q\left(X_{k}\right) \cup\{a\} .
$$

First we assume $k=1$. Then

$$
Q\left(X_{1}\right)_{0} \subset Q\left(T_{1}\right)_{0} \subset Q\left(Y_{1}\right)_{0} \text { and } Q\left(X_{1}\right)_{0} \cup\left\{a, b_{1}\right\}=Q(T)_{0} \subset Q\left(Y_{1}\right)_{0} .
$$


Note that $\# Q\left(Y_{1}\right)_{0}-\# Q\left(T_{1}\right)_{0} \leq 1, \# Q\left(T_{1}\right)_{0}-\# Q\left(X_{1}\right)_{0} \leq 1$ and $b_{1} \notin Q\left(T_{1}\right)_{0}$. Therefore we have

$$
Q\left(Y_{1}\right)=Q\left(X_{1}\right) \cup\left\{a, b_{1}\right\} \text { and } Q\left(T_{1}\right)=Q\left(X_{1}\right) \cup\{a\} .
$$

Next we assume $k>1$ and $(*)$ hold for $k-1$. Then, similar to the case $k=1$, we can check that

$$
Q\left(Y_{k}\right)=Q\left(X_{k}\right) \cup\left\{a, b_{k}\right\} \text { and } Q\left(T_{k}\right)=Q\left(X_{k}\right) \cup\{a\} .
$$

In particular, we have $T_{k}=I_{\left(Q(i) \backslash\left\{b_{1}, \cdots, b_{k}\right\}\right) \cup\{a\}}$.

We now let $T_{r}:=I_{\left(Q(i) \backslash\left\{b_{1}, \cdots, b_{r}\right\}\right) \cup\{a\}}$ and let $Y_{r}$ be a unique direct predecessor of $T_{r}$ with $Q\left(Y_{r}\right)_{0}=Q\left(T_{r}\right)_{0} \cup\left\{b_{r}\right\}=Q\left(T_{r-1}\right)_{0}$. Since $T_{r-1}$ is injective $k\left(Q\left(T_{r-1}\right)\right)$-tilting module, we have $Y_{r} \geq T_{r-1}$.

Note that $I_{i+1}$ is a minimum element of $\bigcap_{X \in e_{1}(i)}\{T \in$ s-tilt $(\Lambda) \mid T \geq X\}$. Therefore, by combining Lemma 3.1, Lemma 3.2, Lemma 3.3, Lemma 3.4 and Lemma 3.5, we get following.

Corollary 3.6. tilt $(\Lambda)$ is determined by poset-structure of s-tilt $(\Lambda)$. In particular, if $\Lambda$ and $\Gamma$ are two finite dimensional basic hereditary algebras and $\rho$ is a poset isomorphism from s-tilt $(\Lambda)$ to s-tilt $(\Gamma)$, then $\rho$ induces a poset isomorphism

$$
\begin{gathered}
\left.\rho\right|_{\operatorname{tilt}(\Lambda)}: \operatorname{tilt}(\Lambda) \simeq \operatorname{tilt}(\Gamma) . \\
\operatorname{REFERENCES}
\end{gathered}
$$

[AIR] T. Adachi; O. Iyama; I. Reiten, $\tau$-tilting theory. to appear in Compos. Math.

[ASS] I. Assem, D. Simson And A. Skowroński, Elements of the representation theory of associative algebras Vol. 1. London Mathematical Society Student Texts 65, Cambridge University Press, 2006.

[ARS] M. Auslander, I. Reiten and S. Smal $\varnothing$, Representation theory of artin algebras. Cambridge University Press, 1995.

[BB] S. Brenner; M.C.R. Butler, Generalizations of the Bernstein-Gelfand-Ponomarev reflection functors. Representation theory, II (Proc. Second Internat. Conf., Carleton Univ., Ottawa, Ont., 1979), pp.103-169, Lecture Notes in Math., 832, Springer, Berlin-New York, 1980.

$[\mathrm{H}]$ D. HAPPEL, Triangulated categories in the representation theory of finite-dimensional algebras. London Mathematical Society Lecture Note Series, 119. Cambridge University Press, Cambridge, 1988.

[HU1] D. Happel; L. Unger, On a partial order of tilting modules. Algebr. Represent. Theory 8 (2005), no. $2,147-156$.

[HU2] D. Happel; L. Unger, On the quiver of tilting modules. J. Algebra 284 (2005), no. 2, 857-868.

[HU3] D. HAPPEL AND L. Unger, Reconstruction of path algebras from their posets of tilting modules. Trans. Amer. Math. Soc 361 (2009), no.7, 3633-3660.

[IT] C. Ingalls; H. Thomas, Noncrossing partitions and representations of quivers. Compos. Math. 145 (2009), no. 6, 1533-1562.

$[\mathrm{J}] \quad$ G. JAsso, Reduction of $\tau$-tilting modules and torsion pairs. arXiv:1302.2709

[RS] C. Riedtmann; A. Schofield, On a simplicial complex associated with tilting modules. Comment. Math. Helv. 66 (1991), no. 1, 70-78.

[U] L. Unger, Combinatorial aspects of the set of tilting modules. Handbook of tilting theory, L.Angeleri Hügel, D.Happel, H.Krause, eds., London Mathematical Society Lecture Note Series 332, Cambridge University Press, 2007.

Department of Mathematics, Nara Women's University, Kitauoya-Nishimachi, Nara CITY, NARA 630-8506, JAPAN

E-mail address: r-kase@cc.nara-wu.ac.jp 